\title{
"Reserva de la Biosfera Cabo de Hornos y Parque Marino Islas Diego Ramírez-Paso Drake" Lupas en Cabo de Hornos - Telescopios en Atacama
}

\author{
RICARDO ROZZI ${ }^{1} \&$ FRANCISCA MASSARDO² \\ 1. https://orcid.org/0000-0001-5265-8726 \\ 2. https://orcid.org/0000-0003-3124-7164
}

Chile se ha consolidado como una plataforma internacional para la investigación astronómica con el establecimiento de grandes telescopios y observatorios para investigar el espacio exterior, el macrocosmos, desde el desierto de Atacama. En los años 1950s, el camino para el establecimiento de los observatorios astronómicos de Chile se inició con expediciones a lomo de mula de (Silva 2019). El año 2000, en el extremo sur de Chile, en el Cabo de Hornos se inició la investigación del "espacio interior", el microcosmos, investigando con lupa líquenes, musgos, algas, invertebrados, aves, y sus interrelaciones a distintas escalas en desde el Parque Omora en Puerto Williams. El 2021, hemos adjudicado el nuevo Centro Internacional Cabo de Hornos (Cape Horn International (enter, (HIC) que ha sido adjudicado como Centro de Excelencia con Fondos Basales de la Agencia Nacional de Investigación y Desarrollo (ANID). CHIC integra las ciencias naturales y sociales, las humanidades, la educación y la ética para apreciar múltiples formas de conocimiento ecológico sobre la biodiversidad y cuidarla (Figura 1). Para investigar desde la escala de nuestro planeta hasta la escala de pequeños organismos, y utilizará la infraestructura del Centro Subantártico Cabo de Hornos que proporciona una plataforma científica para el estudio del cambio global desde el extremo austral de Chile.

En este volumen de la revista Anales del Instituto de la Patagonia damos continuidad a la sección "Reserva de la Biosfera Cabo de Hornos y Parque Marino Islas Diego Ramírez-Paso Drake". Se presenta el descubrimiento de dos especies nuevas para la ciencia mundial: el liquen crustoso, escamoso de color ocre Candelariella magellanica que fue recolectado sobre corteza de árboles de lenga (Nothofagus pumilio), y el hongo sapróbico Sclerococcum nothofagi, también recolectado sobre la corteza de árboles del género Nothofagus (Etayo et al. 2021). Estos descubrimientos y el catálogo de más de 400 especies de líquenes proporcionan nueva evidencia sobre el "hotspot" o "punto caliente" de biodiversidad a nivel mundial para este grupo de organismos que se conserva en la Reserva de la Biosfera Cabo de Hornos (Rozzi et al. 2008). Estos estudios florísticos son el resultado de una larga y fructífera colaboración entre investigadores españoles, liderados por Javier Etayo y Leopoldo G. Sancho, y de la Universidad de Magallanes junto a otros investigadores internacionales 
Fig. 1. Interpretación artística de tres atributos únicos de la Reserva de la Biosfera Cabo de Hornos como laboratorio natural. (1) El mapamundi invertido muestra la ubicación geográfica del Cabo de Hornos como la "cumbre sur de las Américas" que ofrece una región ideal ( $y$ aún poco estudiada) para investigar el cambio global. (2) La lupa de mayor tamaño simboliza el enfoque de investigación biocultural del nuevo Centro Internacional Cabo de Hornos (Cape Horn International Center,

(HIC). (3) La lupa de menor tamaño señala al nuevo edificio del Centro Subantártico Cabo de Hornos que albergará a CHIC, y consolidará dos décadas de investigaciones subantárticas desarrolladas por el equipo Parque Omora - Universidad de Magallanes, Puerto Williams. Concepto y Diseño Ricardo Rozzi; Diseño y Arte Mauricio Álvarez, Archivo Parque Omora.

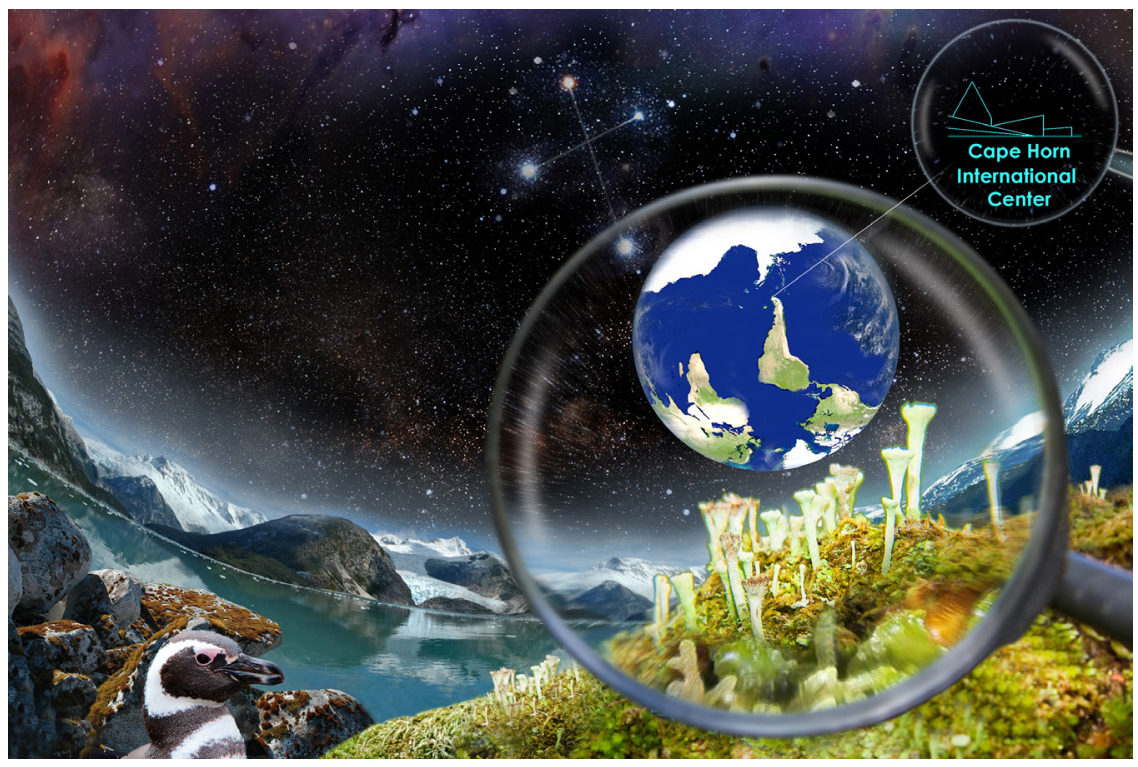

e internacionales. Con el fin de reforzar esta línea de investigación, el Herbario del Instituto de la Patagonia (HIP) ha inaugurado un nuevo catálogo y herbario sobre la micobiota liquénica de la Región de Magallanes y Antártica Chilena que incluye información sobre hábitat, localidades de colecta, distribución en Chile, distribución global y números de los exsiccata (Pineda-Cáceres 2020).

Este número incluye también un novedoso análisis de la variabilidad espacial de los ensambles bentónicos de los ecosistemas intermareales del canal Beagle, con foco en la bahía Yendegaia, Reserva de la Biosfera Cabo de Hornos (Rodríguez et al. 2021). Este análisis identificó seis comunidades con una marcada zonación vertical y variación horizontal. La investigación reveló patrones de distribución a escala espacial fina y discute factores ecológicos que podrían incidir sobre la distribución y abundancia de especies y ensambles bentónicos intermareales en ecosistemas subantárticos de Magallanes. Junto con nueva información sobre la biota y ecología intermareal del canal Beagle, este artículo describe un modelo de estudio con un diseño anidado con diferentes escalas espaciales desde centímetros a kilómetros que podría ser aplicado en otras regiones del país y del mundo.

En los ecosistemas intermareales de la isla Gonzalo en el archipiélago Diego Ramírez se registró un caso excepcional de churrete austral (Cinclodes maculirostris Dabbene, 1917) con una coloración aberrante: plumas blancas en el lomo, flancos y rectrices, pero sus patas, pico y ojos eran de coloración normal (Torres-Mura y Barroso 2021). Esta condición se denomina leucismo parcial y constituye el primer registro de amelanismo para el género Cinclodes. Esta singularidad se suma a la alta diversidad de moluscos y algas costero-marinas del archipiélago Diego Ramírez (Marambio 
et al. 2020, Rossenfeld et al. 2020). Estos resultados han sido posibles gracias a la estrecha colaboración entre investigadores y la Armada de Chile en el sitio de estudios ecológicos a largo plazo isla Gonzalo (Massardo 2020, Rozzi et al. 2020).

A una escala mayor, en esta sección se presenta la primera descripción detallada de los notables gradientes climáticos de la Reserva de la Biosfera Cabo de Hornos (Aguirre et al.2021). Se examinó cómo se interrelacionan estos gradientes con la distribución de ecosistemas y formaciones vegetacionales y determinó que la precipitación es el principal factor que segrega la distribución de los bosques siempreverdes y de bosques mixtos (siempreverdes-deciduos) y/o deciduos. En cambio, para la distribución de los ecosistemas de turberas y praderas la intensidad de los vientos en zonas más expuestas fue el factor climático con mayor influencia. La Reserva de la Biosfera Cabo de Hornos (RBCH) alberga un gradiente de precipitaciones excepcionalmente marcado en distancias muy cortas. Estos ecosistemas subpolares del hemisferio sur han sido mucho menos estudiados que los ecosistemas subpolares del hemisferio norte. Por lo tanto, estos son los primeros resultados que permiten comparaciones de vulnerabilidad de la flora subantártica, especialmente altoandina, con sus floras homólogas del hemisferio norte.

En síntesis, en esta sección se entregan descubrimientos de relevancia mundial para comprender los complejos procesos asociados a al cambio climático global. Esta información provee una base para el nuevo Centro de Excelencia Científico y Tecnológico en un área remota en el extremo sur de Chile que consolidará un apoyo logístico con una instalación de clase mundial en Puerto Williams, ciudad capital de la comuna Cabo de Hornos y de la Provincia Antártica Chilena. De esta forma, Chile se destaca por su ciencia en ambas zonas extremas del país: en el extremo norte con telescopios y observatorios que posibilitan descubrimientos astronómicos en el macrocosmos, y en el extremo sur con lupas (físicas y metafóricas) que posibilitan descubrimientos sobre el microcosmos, que entregan información esencial para modelos de desarrollo que favorezcan la sustentabilidad de la vida y la gestión de las áreas protegidas más australes de América.

\section{AGRADECIMIENTOS}

Agradecemos a la Armada de Chile, Carabineros de Chile, Corporación Nacional Forestal, Dirección General de Aguas del Ministerio de Obras Públicas, Ministerio del Medio Ambiente, Ministerio de Bienes Nacionales, Subsecretaría de Pesca y Subsecretaría de Turismo del Ministerio de Economía, Fomento y Turismo por el apoyo para la implementación de la Reserva de la Biosfera Cabo de Hornos y/o Parque Marino Islas Diego Ramírez - Paso Drake. La preparación de este trabajo y de esta Sección Especial que inauguramos en la revista Anales del Instituto de la Patagonia ha contado con el apoyo de los proyectos Centers de Excelencia con Financiamiento Basal Cape Horn International Center (CHIC-FB210018) de PIA-ANID a la Universidad de Magallanes (UMAG) y del Grupo de Trabajo Mar y Tierra (The Pew Charitable Trust-Chile) a la Fundación Omora. Esta es una contribución del Programa de Conservación Biocultural Subantártica, conjuntamente coordinado por UMAG y University of North Texas (UNT).

\section{LITERATURA CITADA}

Aguirre, F., Squeo, F.A., Casassa, G., Lopez, D., Crego, R., Buma, B., Carvajal, D., Jaña, R. \& Rozzi, R. (2021). Gradientes climáticos y su influyente rol sobre los ecosistemas terrestres de la Reserva de Biosfera Cabo de Hornos, Chile. Anales Instituto Patagonia 49: 1-33. https://doi.org/10.22352/AIP202149012 
Etayo, J., G. Sancho, L., Gómez-Bolea, A., Søchting, U., Aguirre, F. \& Rozzi, R. (2021). Lichens (including some lichenicolous \& corticolous fungi) of Navarino Island, Chile. Anales Instituto Patagonia: 49:1-110. https://doi.org/10.22352/ AIP202149013

Massardo, F. 2020. Reserva de la Biosfera Cabo de Hornos y Parque Marino Islas Diego Ramírez-Paso Drake. Anales Instituto Patagonia 48(3): 39-44.

Pineda Cáceres, J., Morano Büchner, S. \& Vidal, O. J. (2020). Catálogo de las colecciones de líquenes de la región de Magallanes y Antártica Chilena depositadas en el herbario del Instituto de la Patagonia (HIP). In Anales del Instituto de la Patagonia (Vol. 48, No. 1, pp. 7-26).

Rodríguez, J.P., Rosenfeld, S., Bahamonde, F., Rozzi, R. \& Mansilla, A. (2021). Variabilidad espacial de ensambles bentónicos intermareales en Bahía Yendegaia, Canal Beagle, ecorregión subantártica de Magallanes. Anales Instituto Patagonia: 1-24. https://doi.org/10.22352/AIP202149017

Rosenfeld, S., Marambio, J., Aldea, C., Rodríguez, J.P., Méndez, F., Gonzalez-Wevar, C., Gerard, K., Contador, T., Mackenzie, R., Rozzi, R. y Mansilla A. (2020). Actualización del catastro de ensamble de moluscos costero-marinos del archipiélago Diego Ramírez ( $56^{\circ} 31^{\prime} \mathrm{S}$ ), chile: un refugio para la economía sustentable y conservación subantártica. Anales Instituto Patagonia 48(3): 113-125. http://dx.doi.org/10.4067/S0718-686X2020000300113

Rozzi, R., Armesto, J., Goffinet, B., Buck, W., Massardo, F., Silander, J., Kalin-Arroyo, M., Russell, S., Anderson, C.B., Cavieres, L. \& Callicott, J.B. (2008). Changing lenses to assess biodiversity: patterns of species richness in sub-Antarctic plants and implications for global conservation. Frontiers in Ecology and the Environment 6: 131-137.

Rozzi, R., Crego, R.D., Contador, T., Schüttler, E., Rosenfeld, S., Mackenzie, R., Barroso, O., Silva-Rodríguez, E.A., ÁlvarezBustos, X., Silva, A., Ramírez, I., Mella, J., Herreros, J., Rendoll-Cárcamo, J., Marambio, J., Ojeda, J., Méndez, F., Moses, K.P., Kennedy, J.H., Hargrove, E., Russell, S., Goffinet, B., Sancho, L., Berchez, F., Buma, B., Aguirre, F., Sánchez-Jardón, L., Barros, E., Vásquez, R.A., Arroyo, M.T.K., Poulin, E., Squeo, F., Armesto, J.J., Mansilla, A., \& Massardo, F. (2020). Un centinela para el monitoreo del cambio climático y su impacto sobre la biodiversidad en la cumbre austral de américa: la nueva red de estudios ecológicos a largo plazo Cabo de Hornos. Anales Instituto Patagonia 48(3): 45-81.

Silva, B.K. (2020). Stars, Mules, and Interferometers in Early Transnational Astronomy in 1960s Chile. Arcadia, Autumn 2020, no. 40 - Arcadia Collection: Technology and Expertise. (Accesed in December 2021 https://www. environmentandsociety.org/arcadia/stars-mules-and-interferometers-early-transnational-astronomy1960s-chile)

Torres-Mura, J.C., \& Barroso O. (2021). Amelanismo en Cinclodes maculirostris (Furnariidae), Ave Endémica del Extremo Austral de América. Anales del Instituto de la Patagonia 1-5. https://doi.org/10.22352/AIP202149020

Apéndice: Afiliación declara por cada uno de los autores

Número
afiliación Nombre de la institución y/o organización Afiliación

$1 \quad$ Sub-Antarctic Biocultural Conservation Program, departments of Philosophy Eamp; Religion and of Biological Sciences, University of North Texas, Denton, TX, USA.

2 Programa de Conservación Biocultural Subantártica, Universidad de Magallanes, Puerto Williams, Chile;

\begin{tabular}{lc}
\hline \multicolumn{1}{c}{ Autor } & Afiliación \\
\hline Ricardo Rozzi & 1 \\
Francisca Massardo & 2 \\
\hline
\end{tabular}

